\title{
Do dietary patterns change following gastric bypass surgery?
}

\author{
S. Reeves ${ }^{1}$, J. Huber ${ }^{1}$, Y. Wake ${ }^{1}$, E. L. Gibson ${ }^{1}$, B. Rees ${ }^{1}$ and C. Le Roux ${ }^{2}$ \\ ${ }^{1}$ Health Sciences Research Centre, Roehampton University, London SW15 4JD, UK and ${ }^{2}$ The Imperial Weight Centre at \\ Charring Cross Hospital, London W6 8RF, UK
}

Bariatric surgeons have anecdotally reported changes in eating habits of obese people following gastric bypass surgery (GBS), which is believed to be the result of modified food and taste preferences as opposed to restrictions imposed by the bypass itself. Only limited systematic evidence available suggests that consumption of fatty foods is reduced while vegetable consumption increased following GBS $^{(1)}$. This pilot study aimed to compare dietary intakes before and after GBS in a cross-sectional UK sample.

Data were collected from patients at the Imperial Weight Centre in Charing Cross Hospital using a modified 3-d food diary that included questions on postprandial sensations. The diaries were included into routine pre and 8-week post-operative questionnaires. Data are available on twenty-four pre-operative patients and eleven post-bypass patients. Diaries were analysed using Dietplan6 software.

As expected the bypass patients had significantly lower mean energy intakes 7937.04 (SD 3217.49) $\mathrm{kJ} / \mathrm{d}$ in the pre-operative group and $5171.42(\mathrm{SD} 1958.11) \mathrm{kJ} / \mathrm{d}$ in post-bypass group, $(P=0.02)$. Although the number of grams of protein, fat and carbohydrate consumed were significantly lower in the bypass group, the relative contributions to energy intake from the macronutrients were unaltered, $(P=0.4)$. Following surgery it is recommended that the bypass patients take a multivitamin tablet, and as a result the post-bypass patients were consuming significantly more $\mathrm{Cu}, \mathrm{Zn}$, Se, vitamins B, D, E, B1, B2, B3, folate than the pre-operative patients. There were no significant differences in the mean amount of fruit and vegetables consumed between the groups: 2.8 (SD 1.1) and 2.5 (SD 1.2) portions/d for the preoperative and post-bypass groups, respectively. Postprandial sensation data indicated that a third of post-bypass patients reported feelings of nausea, particularly after the consumption of acidic foods or cheese.

This pilot study did not find any change in dietary intake in terms of relative amounts of macronutrient intake following GBS. Sensation of nausea following intake of certain foods deserves further research. Increasing the sample size to provide reliable evidence on taste preferences and food choices of post-operative GBS patients is required, in order to provide appropriate dietetic advice.

1. Olbers T, Bjorkman S, Lindroos A et al. (2006) Ann Surg 244, 715-722. 